\title{
Environmental sensor networks and continuous data quality assurance to manage salinity within a highly regulated river basin.
}

\author{
Nigel W.T. Quinn, PhD, PE, D.WRE \\ HydroEcological Engineering Advanced Decision Support \\ Lawrence Berkeley National Laboratory \\ 1 Cyclotron Road, Bld. 90-1116 \\ Berkeley, CA 94720, USA \\ Ricardo Ortega \\ Grassland Water District \\ 22759 S. Mercy Springs Road \\ Los Banos, CA 93635 \\ Lisa M. Holm, PE \\ Bureau of Reclamation \\ Division of Planning, 2800 Cottage Way, MP-700 \\ Sacramento, CA 95825
}

\begin{abstract}
Environmental sensor networks enjoy widespread deployment as monitoring systems have become easier to design and implement in the field and installation costs have fallen. Unfortunately software systems for data quality assurance have not kept pace with the development of these sensor network technologies and risk compromising the potential of these innovative systems by making it difficult to assess the accuracy and consistency of the data. Lingering uncertainty can constrain the willingness of stakeholders to make operational decisions on the basis of the real-time sensor data - a few negative experiences can do irreparable damage to a project which is attempting to change stakeholder behavior. Management of river salt loads in complex and highly regulated river basins such as the Murray Darling Basin in south-east Australia and the San Joaquin Basin in California, USA present significant challenges to Information Technology infrastructure within resource agencies that often have a poor history of coordination and data sharing. In the San Joaquin Basin - web-based environmental data dissemination initiatives to address salinity issues need to overcome a fear of loss of autonomy as well as data quality assurance and data reliability issues. These environmental decision support issues are contrasted with those facing resource managers in the Murray Darling Basin. This paper describes a new approach to environmental decision support for salinity management in the San Joaquin Basin of California that focuses on web-based data sharing using YSI Econet technology and continuous data quality management using a novel software tool, Aquarius. Commercial turn-key monitoring systems such as YSI EcoNet provide real-time web-access to sensor data as well as providing the owner full control over the way the data is visualized. The same websites use GIS to superimpose the monitoring site locations on maps and local hydrography and allow point and click access to the data collected at each environmental monitoring site. This Information Technology suite of software and hardware work together to provide timely, reliable and high quality data in a manner that can used by stakeholder decision makers to better manage salt export to the San Joaquin River and ensure compliance with State water quality objectives. The technologies developed for this application can be extended to improve compliance with TMDL water quality objectives over entire river basins and should have applicability in any watershed where environmental decision support systems are being developed to assist for stakeholders as part of a coordinated strategy for non-point pollutant load reduction
\end{abstract}

Keywords: TMDL, sensor networks, real-time water quality monitoring, seasonal wetlands, quality assurance, salt load management 


\section{Introduction}

In the past five years there has been a revolution in the way individuals share information. Social networking software applications such as Facebook, MySpace, and YouTube continue to redefine the manner by which knowledge is acquired and shared. The US Environmental Protection Agency Office of Information Technology has suggested that the next five years will witness as significant a technical revolution in data sharing technologies as the past five have provided in the ability to search. As data sharing becomes more widespread a significant constraint to the use of the information acquired is the issue of data quality. Important decisions are often made on current or real-time data which is why, in the past, agencies preferred to establish and maintain their own monitoring stations. However, scant attention and few resources have been devoted to sharing the data from these stations with others (Maidment, 2008; Tarboton, 2005).

The Consortium for the Advancement of Hydrologic Science (CUAHSI) has developed a Hydrologic Information System architecture in an attempt to address some of the obstacles associated with data sharing on the web (Maidment, 2008). Given the rapid increase in statutory environmental regulation during the past decade and the establishment of pollutant-load regulatory frameworks in many countries such as the TMDL (Total Maximum Daily Load) in the United States (CEPA, 2002), stakeholders are being obligated to support the extensive monitoring networks needed to implement these pollutant control systems. Data sharing technologies and procedures for ensuring continuous data quality assurance are necessary for sustained and cost-effective watershed-based pollutant regulation. This paper describes a prototype data acquisition, sharing and data quality assurance project developed for salinity management within the San Joaquin Basin of California (Figure 1) that takes a first step at overcoming existing impediments. The paper provides detail on wetland salinity management since the 80,000 hectare project area to which these techniques are applied was without any form of continuous environmental monitoring as recently as six years ago. The salinity control strategy taken to manage salt exports from these San Joaquin Basin wetlands is contrasted with the Basin salinity plan adopted for the significantly larger Murray Darling Basin in south-east Australia to contrast and compare the relative merits of each program.

\section{Salinity management in the Murray Darling Basin}

Salinity management is an important sustainability issue in arid river basins around the world especially those that support an agricultural sector reliant on irrigated agriculture. A number of simulation models and environmental decision support systems have been developed for river basin and irrigation management (Fedra, 1993; Zu and Dale, 2001 and Garcia et al, 2000) or wetland management (Vadas et al., 1995; Janssen et al., 2005) that have helped to rationalize data collection activities and improve systems thinking. However none of these studies has attempted to integrate monitoring, modeling and visualization into a single environmental decision support system. The Murray River Basin in south-east Australia and the San Joaquin Basin in California (Figure 1) require integrated information technology, monitoring and modeling to support regulatory Basin Plans for salinity management. Salinity management within the Murray Darling Basin in Australia is perhaps the most evolved in the world. In the Murray-Darling Basin salt loads are administered as part of a market-based licensing and trading system by a central management authority (Murray Darling Basin Commission, 2005; Murray-Darling Basin Ministerial Council, 2001.). This system allows parties to buy and sell permits for salt load export or credits for reductions in salt load export. This system also sets limits on salt loads emitted by holders of environment protection licenses and links licensing fees to salt load exports. (http://www.environment.nsw.gov.au/licensing/ hrsts/allocating. htm). A website is used to communicate to stakeholders and educate the public on the elements of the licensing program, the basis for allocation of permissible salt loads and the rules for salt load trading. The licensing system combines the strengths of several water quality regulatory instruments to achieve optimal environmental and economic outcomes. Features of the system are that it: (a) sets a clear set of minimum standards for environmental performance; (b) incorporates incentives for salt load reduction; (c) gives licensees the flexibility to implement innovative cost-effective salt load abatement methods; (d) provides the infrastructure for salt load trading and ability to calculate salt load using a defined Load Calculation Protocol.; (e) enables the long-term tracking of salt load reductions; (f) 
requires each licensee to submit an annual return at the end of each 12-month license fee period. Under this system stakeholders in the Murray Darling Basin that discharge pollutants must obtain sufficient tradable units to compensate for their salt load export. Stakeholders that reduce salt loads may have surplus salt load credits that they can sell to others that find emission reduction more expensive or difficult.

\section{Salinity management in the San Joaquin Basin}

Salt export from agricultural, wetland and municipalities in the San Joaquin Basin is regulated as part of a comprehensive Total Maximum Daily Load (TMDL) (CRWQCB, 2004). The TMDL is intended to identify, quantify and help control sources of pollution that affect attainment of water quality objectives and full protection of identified beneficial uses of water. The TMDL includes both point and non-point sources of salt load. Point sources of salinity, such as discharges from wastewater treatment systems, are regulated using Waste Load Allocations (WLA). These WLA's are typically concentration based and allow the entities regulated to enter into a marketplace with other regulated entities to trade their allocations. Non-point sources of salinity (LA) are not typically amenable to the establishment of fixed monthly or seasonal salt load allocations because of the diffuse nature of these non-point source loads in the watershed which makes it difficult to assign responsibility, the technical challenges of monitoring individual discharge points and the high seasonal variation in export flows and salt loads. Base salt load allocations (LC) are made to account for the variable assimilative capacity for salt within the San Joaquin River - these are calculated based on the lowest anticipated flow condition in the River and the River's assimilative capacity (LC) for salt load during these episodes. Point source (WLA's), background salt loading and salt loads contained in groundwater return flows to the River are subtracted from the total assimilative capacity of the River to determine the salt load allocation to all non-point sources.

$$
\begin{aligned}
\text { TMDL }=\text { ALC } & =\Sigma W L A+\Sigma L A+\text { MOS } \ldots \ldots \ldots \ldots \ldots(1) \quad(C R W C B, 2004) \\
\text { Where }: \text { LC } & =\text { salt load capacity (tonnes of salt) } \\
\text { WLA } & =\text { point source salt load allocation (tonnes of salt) } \\
\text { LA } & =\text { non-point source salt load allocation (tonnes of salt) } \\
\text { MOS } & =\text { margin of safety (\%) }
\end{aligned}
$$

The margin of safety is typically set at $15-20 \%$ of the total salt load and takes account of the hydrologic variability of the system and the technical inability to use $100 \%$ of the assimilative capacity of the River, even under near-perfect management.

In the San Joaquin Basin most of the irrigation water supplied to the west-side of the San Joaquin Basin is pumped from the Sacramento-San Joaquin Delta and contains low levels of salinity (usually between 300 and $450 \mathrm{ppm}$ ) - hence allowances have been made for the salt load contained in imported water supply and for the evapoconcentration of this water when consumptively used by crops or delivered to seasonal wetlands. The TMDL (CRWQCB, 2004) recognizes a water supply salt load credit for the salinity of water imported from the Delta and a consumptive use allocation credit for agricultural and wetland use of the water (an incentive for these entities to discharge return flows that have not been degraded).

The salinity TMDL for the San Joaquin Basin is unique in that it permitted an additional "real-time" load allocation that supercedes the conservative base non-point source load allocation (LA), provided a real-time salinity management program is implemented in the San Joaquin Basin. The core requirements of this program include : the development of a sensor network to perform real-time monitoring of flow and salinity data for stakeholders from contributing watersheds; a data dissemination system for effective sharing of data among basin stakeholders to allow forecasting of River assimilative capacity; and sanctioning by the Regional Water Quality Control Board. The impact of this additional real-time salt load allocation would be to permit significantly larger export of 
salt load from the watershed - helping to overcome salt build-up within the shallow groundwater system which would ultimately degrade the groundwater resource within the Basin.

The TMDL is a convenient regulatory tool that encourages the trading of pollutant credits - where the ability to pollute has an economic value and can be traded amongst dischargers to receiving waters of the State. Failure to meet State water quality standards or numerical water quality objectives is typically penalized through the imposition of a fine that is typically assessed in proportion to the magnitude of the exceedence. In this system the market place is expected to determine the equilibrium point where the demand for and supply of pollutant credits are equated. However, developing a marketplace for non-point source salt loads is more difficult than for point source salt loads because of the inherent difficulty in matching sources with discharges over large regions. Unlike the Murray Darling Basin where salt loading is regulated to allow flexibility of operation the San Joaquin Basin is over-constrained - TMDL's for other constituents such as dissolved oxygen conflict with the goal of reducing salt loading in the San Joaquin River. Federal regulations for power generation along the tributaries to the San Joaquin River and minimum prescribed in-stream flows to protect an anadromous fishery further limit the opportunities offered by real-time salinity management during certain months of the year. Further impediments to the development of a fully-functional trading program for non-point source salt loads are created by the "phased" approach being followed, which limits the number of players in the marketplace seeking credits in the early stages of the program.

The success of the TMDL concept as a regulatory tool is testimony to the consistency of the methodology and its ability to provide quantitative measures of application outcome. The conceptual TMDL model breaks down in regions such as the western United States where hydrology is often extreme, with wet hydrologic years, capable of causing severe flooding, interspersed with periods of severe drought - which can cause great stress on the water delivery system. Another limitation of the TMDL approach, compared with the more adaptive salt load accounting system in the Murray Darling Basin, is the inflexibility of the methodology to account for non-standard watershed characteristics or systems where watershed hydrology is subject to adaptive change. Application of the TMDL to management of saline wetland drainage is one of these instances.

\section{Real-time salinity management}

Real-time salinity management (RTSM) is a strategy for meeting downstream water quality objectives by improving coordination of upstream contaminant loading from non-point sources with dilution flows so as to maintain river assimilative capacity (Quinn and Karkoski, 1998; Quinn and Hanna, 2003). This is a more ambitious and more dynamic regulatory policy than the salinity trading system implemented in the Murray Darling Basin in that, fully realized, it requires active participation of stakeholders throughout the entire Basin. The concept of RTSM relies on the continuous computation and forecasting of River assimilative capacity and information flow between stakeholders - those that supply assimilative capacity and those that consume it. In the case of the San Joaquin Basin east-side reservoir releases from tributaries carrying snow-melt from the granitic Sierra Nevada mountains and return flows from east-side irrigation districts provide dilution to a San Joaquin River that drains westside salt laden soils. Salt loads from water imported from the Delta combine with native salts leached from soils (that were once an uplifted seabed) that comprise west-side drainage return flows. Westside agricultural return flows are highest during the summer irrigation season. Seasonal wetland salt loads are highest during the months of March and April when the majority of the seasonal wetland ponds are drawn down to promote establishment of moist soil plants and other native grasses that provide a protein source to overwintering waterfowl.

Real-time salinity management (RTSM) improves compliance with SJR salinity objectives by improving the coordination of west-side agricultural and wetland dischargers of salt with reservoir releases flows made along east-side tributaries. Hence RTSM is a concept that relies upon access to real-time flow and electrical conductivity data from networks of sensors located along the SJR and its major tributaries (Quinn and Karkoski, 1998). RTSM involves comprehensive watershed flow and water quality monitoring, modeling, salt assimilative capacity forecasting, information dissemination and sharing as part of an Environmental Decision Support System (EDSS). RTSM provides timely 
decision support to agricultural water districts and seasonal wetland managers - allowing them to improve the coordination of salt load export with the available assimilative capacity of the San Joaquin River (Quinn and Hanna, 2003). Although salinity management has been practiced within west-side San Joaquin River Basin agricultural water districts for more than 100 years and salinity management has only become a concern to seasonally managed wetlands since the announcement of the salt TMDL - progress towards RTSM is more advanced in the wetland areas. This is largely because of the more serious long-term consequences of restricted salt export in the seasonally managed wetlands. This paper focuses on implementation of RTSM in San Joaquin Basin seasonal wetlands.

Real-time salinity management applied to seasonal wetlands in the San Joaquin Basin could require alteration to the traditional wetland drawdown schedule. Seasonally managed wetlands in the western San Joaquin Basin of California's Central Valley provide overwintering habitat for migratory waterfowl and hunting opportunities during the annual duck hunting season. Wetland water supply is supplied from the Sacramento - San Joaquin River Delta and contains inorganic salts which evapoconcentrate in man-made, seasonally managed ponds, before being drained (between late March and early May) into channels that discharge into the San Joaquin River (Figure 2). This seasonal wetland drainage, produced within a 80,000 hectare wetland Grasslands Ecological Area (GEA) of the San Joaquin Basin (Figure 2) must be eliminated to preserve salt balance and sustain habitat conditions that make these wetlands the most important migratory bird resource in the western United States. The seasonal wetland drainage schedule typically coincides with the germination period of salt sensitive agricultural crops, irrigated with water pumped from the River downstream by riparian stakeholders. During this drawdown period the assimilative capacity of the River can be exceeded by the wetland contribution leading to negative river assimilative capacity (Figure 3).

Curtailing wetland drainage during this critical period can result in loss of forage value for overwintering waterfowl and increased salt accumulation in shallow wetland soils which can reduce germination success of valuable moist soil plants such as swamp timothy in subsequent seasons.

This drawdown schedule is typically established to promote the establishment of optimal moist soil plant habitat. Changes to this traditional schedule come at a potential cost to the sustainability of the moist soil plant habitat resource (Frederickson and Taylor, 1982, Quinn et al. 2005). There is a dearth of literature upon which to base salinity management strategies primarily because until relatively recently (past six years) there was no continuous monitoring of flow or water quality in to or out the refuges or individual ponds upon which to establish water balances. Wetland managers were able to perform most water management tasks using once-daily samples of flow and water quality. The threat of future regulation of wetland salt exports, combined with a recognition that a lack of data could leave these entities vulnerable to unsubstantiated perceptions of their salt load contribution to the SJR, has created an opportunity to embrace new technologies in web-based, environmental decision support. Recent advances in data sharing technologies such as YSI-EcoNet provide rapid, relatively inexpensive monitoring station deployment and web-access to real-time data. This has provided wetland managers with new tools to understand the relationship between their wetland salt exports and water quality conditions within the SJR.

\section{Data sharing technologies}

Web delivery of the processed water quality data through YSI-EcoNet was initiated in 2005. YSIEcoNet integrates sensor hardware (acoustic flow probes, pressure and water quality sondes) and dataloggers with software that perform local data storage, telemetry and visualization. YSI-EcoNet comprises a mesh of Data Nodes that collect data from flow and water quality measuring sensors and Access Nodes that have the added capability of collecting data via a low power radio interface from surrounding Data Nodes (Figure 4). The Access Node transmits logged data to a remote DataCenter via CDMA cellular phone or satellite modem from which the data is made accessible through the Internet. A remote data storage and processing facility - the NIVIS Data Center - maintains all data collected by the monitoring network at 15 minute intervals through a service contract with YSI Inc. The wireless mesh network topology allows "point-to-point" or "peer-to-peer" connectivity and an ad 
hoc, multi-hop network. The mesh network is self-organizing and self-healing - hence loss of one or more nodes does not necessarily affect its operation. This increases the overall reliability of the system by allowing a fast local response to critical events in the rare event of a communication problem. Elimination of tedious data acquisition and processing procedures through adoption of YSIEcoNet has worked well with our wetland manager client base by eliminating much of the tedium of downloading and processing environmental data. It has also allowed wetland managers more time to perform bi-weekly sensor data quality assurance checks including cleaning of sensors and checking the accuracy of staff gauge data (used in the computation of flow). Wetland managers have appreciated the time saving benefit of reviewing monitoring site data ahead of routine quality assurance checks - this helps prepare field staff for contingencies such as sensor failure prior to travelling to the site. The project monitoring sites are up to $30 \mathrm{~km}$ apart.

\section{Data quality control and assurance}

A limitation of many environmental decision support projects that rely on the telemetry of monitored data to a stakeholder community is data quality assurance. Data quality assurance protocols established for discrete environmental sampling is well established and data quality control plans are integral components of most environmental monitoring projects. However with continuously recorded and reported data - the logistics of monitoring site visitation, data management, processing and error correction become more onerous. Until recently few software tools existed to facilitate and guide these tasks - even the idea of migrating preliminary environmental data to another internet-accessible location after data quality assurance had been performed has not been routinely practiced. Inaccurate or absurd data posted to a project website can cause irreparable harm to a project and can quickly lead to a loss of confidence in the stakeholder community. In our wetland salinity management project cumulative flow calculations which were performed by our acoustic Doppler instrument were accurate when downloaded from the data collection platform but were 100 times too high when transmitted using the SDI-12 protocol to an external datalogger. This turned out to be a programming error in the firmware - a decimal point was omitted in the program and re-inserted during output processing. This error was caught by one of our important project cooperators, who had become a routine user of project real-time data. Our close working relationship prompted early feedback from this cooperator and relatively quick recognition and resolution of the problem. This is not typical problems such as these often fester within the stakeholder community before coming to light - by which time enthusiasm for the project and trust between project proponents and the stakeholder community has been eroded.

\section{Software tools for continuous data quality assurance}

The Aquarius Environment Toolbox (Aquatic Informatics, Inc., 2009) is a suite of software object modules used to manage the entire data processing workflow from the project flow and water quality monitoring stations (Figure 5). The software object modules relevant to the wetland salinity management project include those responsible for: Data Pre-Processing; Data Correction; Modeling; and Output Visualization and Reporting. The Aquarius Whiteboard environment is used to assemble the sequence of object modules that comprise the data processing workflow (Figure 5).

The Data Pre-Processing module comprises software objects for Signal Joining and Signal Trimming. The Signal Joining object is used to append multiple time series data files during each data download so that a complete data record can be compiled within a local database. Storing the data in a local database after performing data quality assurance allows the certified data to be uploaded routinely to the NIVIS Data Center, which previously provided access to uncensored real-time data. The Signal Trimming object is used to trim outliers and periods of incomplete data records from the data record before being stored in the database. At the beginning of the project technical problems such as bad circuit boards and poorly sealed acoustic Doppler sensors produced periods of incomplete or suspect records which had to be removed from the data record. 
The Data Correction object is used to apply manual adjustments to time series datasets. Autonomous collection of data results in anomalies such as biases, drifts, outliers, and non-physical data. Hence, the Data Correction object is used to apply corrections for sensor drift (not typically a problem with the YSI and MACE instruments) or sensor fouling (a significant problem in the wetland environment of the project). For example a Fouling Drift correction is used routinely to compensate for biological fouling or scaling of the water quality sensor that tend to skew readings in a progressive fashion as the biological film or scale develops. Standard practice for quality assurance is to take a single measurement from a sensor immersed in a control solution both before and after cleaning. A more time and cost effective protocol, practiced in the current project, was to calibrate a portable YSI sonde (comprising EC, temperature and pressure sensors) in the laboratory before and after each day in the field. Then the sonde at each monitoring site was calibrated to the ambient portable sonde sensors readings reading at each monitoring site. The sonde EC sensor was recalibrated if the readings differ from the value of the standard solution. Pressure transducers in both the water quality sonde and acoustic Doppler instrument remained stable requiring little adjustment. Monthly removal of biological growth surrounding the pressure transducers was sufficient to keep the transducers from drifting - except in the instances of catastrophic failure where improper sealing with epoxy allowed moisture migration into the sensor.

In the Aquarius software each correction made to the time series data is recorded in the Correction History Manager which logs the information about the correction and makes an entry into the correction history (Aquatic Informatics, 2009). Comments describing the correction appear in small text that is auto generated and can be modified, if necessary, to increase the amount of information for a subsequent correction audit. Offset adjustments were made to correct for a constant (throughout time) bias in the target time series data. Offset adjustments were necessary when a sonde had to be replaced or a sonde moved higher within the stilling well as a result of sediment accumulation around the base of the stilling well. This accumulation of sediment affected water circulation around the electrical conductivity and temperature sensors. Within the Aquarius software the Mark Region tool is used to select a section of the target time series where the offset correction can be used to adjust readings upwards or downward. The percent correction feature in the correction control pane of the software allows the user to apply a calculated known percentage correction to the signal at either the beginning of the data sequence or at the end. Data outliers can be removed using these tools and short data gaps can be interpolated without resorting to a model based correction approach. Data gap interpolation controls provide options that include the application of a linear spine, which simply draws a straight line in between the endpoints of the gap or a cubic spline, which uses up to 5 data points outside the range of the marked gap to guess at the curvature of the time series and using a best-fit curved line. The automated interpolation control options within the Aquarius toolbox are a significant technical advance over existing software and are critical for the anticipated expansion of existing sensor networks necessary to fully implement RTSM. Manual as well as automated interpolation changes are all noted in the Correction History Manager. Each time a correction is applied to a section of target time series data the Correction History Manager is invoked to capture details about the user performing the correction and the rationale for performing the correction.

The Modeling object within the Aquarius toolbox has proved useful in the application of calibration factors to discharge data derived from acoustic Doppler velocity and pressure sensors. Acoustic Doppler velocity sensors sample the flow volume either approaching or moving away from the sensor. In open channels with fluctuating stage or in culverts which can flow partially full - it is difficult to obtain a true mean area-velocity owing to the friction along the perimeter of the conveyance structure. Flow rating relationships between reported and actual flow have been developed for each monitoring site and the rating equation built into the modeling object to transform the reported flow value to an actual flow. At a number of monitoring sites weir boards (flash board risers) provide the means of controlling wetland pond stage and pond outflow - at these sites the modeling object was first used to calculate discharge using the weir equation which is a function of board width and height and water stage over the weir boards. However the difficulty of maintaining continuous records of weir board adjustments proved too onerous for wetland managers and became a bottle-neck for real-time data 
reporting. Acoustic Doppler flow sensors were retrofitted at each wetland drainage site to address this issue.

The Aquarius toolbox contains a full suite of Output Visualization and Reporting tools (Figure 5) that allow easy review and auditing of any data sequence within the time series database at each monitoring site. The Quick View object displays time series data in a spreadsheet format which facilitates plotting and charting and can be used to provide descriptive statistics of each sensor time series record. It has been used to create overlay multiple data traces within a single window in order to check the functioning of suspect sensors in the monitoring network as part of the data quality assurance program. Reporting the quality certified data back to the NIVIS website (Figure 6) was a feature initially lacking in the commercial release of YSI EcoNet. The NIVIS Data Center did not permit client access to the data except as served through the NIVIS EcoNet website - rather the Data Center provided a means of discriminating between public and private (password protected) access to the website. This had the unfortunate consequence of restricting public access to current data only, given our cooperators reluctance to share potentially flawed data with the general public, as discussed at the beginning of this paper. Only project personnel and cooperators were provided with private user access allowing these clients to download the time series data record. In late 2009 a solution was negotiated between YSI Inc. and the NIVIS Data Center that provided a data export wizard and the ability to add a second data column paired with each of the web- reported sensor outputs. The schema for the protocol appears in Figure 6. Under this protocol public access clients can view all historic data once they have been migrated into the second column after having undergone data quality assurance procedures. Private access clients are able to view both columns of data on the project website and have full access to the time series data for all monitoring sites. Automation of the data filtering and web posting process will speed-up stakeholder access time to information needed to make RTSM decisions. These actions will help develop the assurances needed for stakeholders to fully implement RTSM within the San Joaquin River Basin.

\section{Water quality forecasting and decision support}

Successful implementation of RTSM in the San Joaquin Basin requires that salt loads exported from managed wetlands and other west-side dischargers within the Basin never exceed the calculated assimilative capacity of the San Joaquin River. River assimilative capacity (expressed as a salt mass load) is determined by the product of river flow and the ambient water quality standard for salinity, established at the downstream compliance monitoring station. A decision support tool was developed to simulate daily river assimilative capacity and permit forecasting of allowable salt loading from managed wetlands in the Basin based on real-time flow and salinity data.

The Watershed Management Framework (WARMF) model (Herr et al., 2001; Chen et al., 2001, Herr and Chen, 2006; Keller, 2000) is comprehensive decision support tool specifically designed to facilitate TMDL development at the watershed-level. The WARMF-SJR application simulates the hydrology of San Joaquin River Basin and performs mass balances for a broad suite of potential contaminants including total dissolved solids. The model (Figure 7) simulates tributary inflows from the major east-side rivers, agricultural and wetland drainage return flows, riparian and appropriative diversions and uses hydrologic routing to calculate flow and water quality at approximately $1 \mathrm{~km}$ intervals along the main stem of the SJR. Wetland drainage from the Grassland Ecological Area was partitioned into component State, Federal and private wetland contributors to SJR salt load. A GISbased graphical user interface (GUI) facilitates the visualization of model input flow and water quality data. Data templates expedite automated data retrieval from State and Federal agency hydrology and water quality databases and the automated updating of model input files. Water managers can enter daily schedules of diversions and discharges using the spreadsheet formatted model data interface. Standardized model output graphics aid the dissemination of flow and water quality forecasts.

A wetland water quality model has been developed that simulates water and salinity balances for individual ponded areas aggregates wetland discharge and salt loading at the watershed level enabling comparison with, yet to be established, salt loading targets for the Grasslands Ecological Area. The 
model is similar in design to models of irrigated agriculture under saline conditions (Mahmoud and Garcia, 1995; Garcia and Lange, 1993; Vadas et al., 1995) except for the fact that wetlands are flooded in the fall and drained in the spring. Monthly sub-basin, seasonally-adjusted salt load targets are one of a number of salinity management strategies that could be used to encourage cooperation and coordination of wetland drawdown by State, Federal and private wetland stakeholders. Coalition building between stakeholders with like interests and who are required to comply with similar environmental regulations has been effective in California as a means of retaining local control and management flexibility. The WARMF model is being used as the general simulator for the wetland decision support system application. http://www.epa.gov/ATHENS/wwqtsc/html/warmf.html . The new model application will track the salt concentration within each ponded area based on estimated wetland evaporation, seepage and cumulative inflow and outflow and the salinity concentrations of these fluxes. Output from the WARMF wetland model application will overwrite estimated values for the State, Federal and private wetland salt loads in the WARMF-SJR model - to provide a more accurate assessment of San Joaquin River assimilative capacity.

\title{
Institution building
}

Realization of the potential of RTSM will require the formation of a watershed-level salinity management entity with the authority to encourage compliance with sub-basin salt load targets and to impose penalties for violation of these established salt load limits. A local coalition of entities discharging drainage to the San Joaquin River formed a "Drainage Authority" to coordinate flow and water quality monitoring requirements imposed by the Regional Water Quality Control Board (a State institution responsible for implementation of TMDL's in the San Joaquin River Basin). This Drainage Authority is financed by stakeholder contributions and retains a staff to oversee the activities of the agricultural, wetland and municipal dischargers of salt and other contaminants into the San Joaquin River. Oversight involves maintenance of monitoring stations, responsibility for discrete sample collection and analysis and monitoring data record keeping. Implementation of RTSM will require a similar institution though with added responsibilities of coordinating both drainage return flows and reservoir releases from east-side tributaries, synthesis of real-time data and dissemination of daily salt assimilative capacity forecasts for the San Joaquin River. At the present time a conceptual design of this new institution has not been formulated.

\begin{abstract}
Summary
Technical advances in data acquisition and information dissemination technologies have made possible the implementation of a real-time salinity management program in California's San Joaquin Basin. Experience to date with respect to implementation of RTSM in the San Joaquin Basin suggests the following principles that have been discussed in the paper: (a) necessary stakeholder participation in the design and operation of environmental monitoring stations; (b) decision support system design features need to recognize the time constraints, technical expertise and work preferences of those responsible for implementing RTSM; (c) simulation models that form the core of environmental decision support systems should have map-based interfaces, retrieve real-time data through automated web browsing tools and provide visualization designed to aid basin stakeholder understanding and the consequences of management decisions; (d) new commercial technologies that promise to improve measurement accuracy and reliability and streamline environmental decision support often bring along their own unique problems and challenges. Software bugs and hardware malfunctions are common and early adopters are advised not to over-promise outcomes, at least in the early stages, while these problems are being addressed. (e) sensor web technologies such as YSIEcoNet and powerful signal processing software such as Aquarius have provided a quantum leap toward successful implementation of RTSM
\end{abstract}

The process of implementing RTSM will further encourage innovation - successful implementation will have significant transfer value to other highly regulated river basins where water quality is a concern. 


\section{Acknowledgements}

The authors would like to thank colleagues within the Bureau of Reclamation, California Department of Water Resources and Central Valley Regional Water Quality Control Board who have funded research projects over the past decade directed at implementing real-time water quality management in the San Joaquin Basin of California. Thanks also to John Beam, California Department of Fish and Game, and both David Widell and the late Don Marciochi, Grassland Water District who have allowed the extension of the general concepts of RTSM to the managed seasonal wetlands resource in the Grasslands Ecological Area. Rick Ortega, Lara Sparks and Patrick Rahilly have provided excellent project support. Thanks also to Aaron Rollo, Tim Finnegan and David Lee and from YSI Inc. who have provided valuable technical support and who have chosen our current wetland salinity management project as a showcase for the YSI-ECONET technology in California.

This work was partially supported by the U.S. Department of Energy under Contract No. DE-AC02$05 \mathrm{CH} 11231$.

\section{References}

Aquatic Informatics Inc., 2008. Aquarius. Help Manual release 2.3. Vancouver, BC, Canada. http://info@aquaticinformatics.com

California Environmental Protection Agency, 2002. Total Maximum Daily Load for Salinity and Boron in the Lower San Joaquin River. Staff report by the Regional Water Quality Control Board, Central Valley Region.

California Water Quality Control Board, 2004. Amendments to the Water Quality Control Plan for the Sacramento River and San Joaquin River Basin. Draft Final Staff Report and Technical TMDL report, Sacramento, CA.

Chen, C.W., Herr, J., and Weintraub, L.H.Z. 2001. "Watershed Analysis Risk Management Framework: Update One: A Decision Support System for Watershed Analysis and Total Maximum Daily Load Calculation, Allocation, and Implementation,” EPRI, Palo Alto, CA. Topical Report 1005181.

Fedra K. 1983. Interactive water-quality simulation in a regional framework: a management-oriented approach to lake and watershed modelling. Ecol. Modelling 21,4 (1983), p. 24.

Fredrickson, L.H. and T.S. Taylor 1982. Management of seasonally flooded mpoundments for wildlife. Resource Publication 148. U.S. Dept. of the Interior, Fish and Wildlife Service, Washington, D.C.

Garcia, L.A., Elhaddad, A., and Patterson, D. 2000. Modeling Irrigation and Drainage System for Drainage Water Quality Management, presented at the International Challenges Facing Irrigation and Drainage in the New Millennium, June 20-24, 2000, Ft. Collins, CO.

Garcia, L.A., and Lange, R. 1993. Capability Assessment of the Integrated River Basin Environmental Management (IREM) Model for Application to the Kendrick Project", Integrated Decision Support Group, Technical Publication, October 1993.

Herr, J., Weintraub, L.H.Z., and Chen, C.W. 2001. "User's Guide to WARMF: Documentation of Graphical User Interface,” EPRI, Palo Alto, CA. Topical Report.

Herr J. and C.W. Chen. 2006. San Joaquin River Model : Calibration Report. CALFED Project ERP02D-P63 Monitoring and Investigations for the San Joaquin River and Tributaries Related to Dissolved Oxygen. Systech Water Resources Inc. San Ramon, CA.

Janssen R, H. Goosen, M.L. Verhoeven, J.T.A. Verhoeven, A.Q.A Omtzigt and E. Maltby 2005. Decision support for integrated wetland management. Environmental Modelling \& Software, Vol. 20, No. 2, pp 215-229

Keller A., 2000 "Peer review of the watershed analysis risk management framework (WARMF): an evaluation of WARMF for TMDL applications by independent experts using USEPA guidelines", Electric Power Research Institute, Palo Alto, CA. Technical Report 1000252.

MACE USA LLC. http://macemeters.com/ P O Box 7144 Shawnee Mission - Kansas City Kansas 66207. 
Mahmoud, M., and Garcia, L.A. 1995. Multi-criteria Decision Support System Implementation and Application, presented at the First International Conference on Multiple Objective Decision Support Systems for Land, Water and Environmental Management: Concepts, Approaches, and Applications, July 23-28, 1995, University of Hawaii, Honolulu, Hawaii.

Maidment, D.R., ed. (2008), CUAHSI Hydrologic Information System: Overview of Version 1.1, Consortium of Universities for the Advancement of Hydrologic Science, Inc, Washington, DC, 96p, http://his.cuahsi.org/documents /HISOverview.pdf.

Murray-Darling Basin Commission, 2005. Strategic Plan 2005-2010. MDBC, Canberra, ACT.

Murray-Darling Basin Ministerial Council, 2001. Basin Salinity Management Strategy 2001-2015. MDBC, Canberra, ACT.

Quinn, N.W.T. and W.M. Hanna, 2003. A decision support system for adaptive real-time management of seasonal wetlands in California. Environmental Modelling and Software, Volume 18, Issue 6, pp. 503-511

Quinn, N.W.T. and J. Karkoski, 1998. Real-time management of water quality in the San Joaquin River Basin, California. American Water Resources Association, Vol. 34, No. 6.

Quinn N.W.T., J.S. Hanlon, J.R. Burns, C.M. Taylor, T Lundquist and W.T. Stringfellow. 2005. RealTime Water Quality Monitoring and Habitat Assessment in the San Luis National Wildlife Refuge. Lawrence Berkeley Laboratory Topical Report \# 58813, Berkeley, CA.

Tarboton, D.G., (2005), "Review of Proposed CUAHSI Hydrologic Information System Hydrologic Observations Data Model." Utah State University. May 5, 2005. http://www.engineering.usu.edu/dtarb/HydroObsDataModelReview.pdf

Vadas, R. G., Garcia, L. A, Labadie, J.W. (1995), "A Methodology for Water Quantity and Quality Assessment for Wetland Development", Water Science and Technology 31(8):293-300.

Yellow Springs International Inc. 2007. https://www.ysi.com/ 1700/1725 Brannum Lane Yellow Springs, OH 45387-1107, USA.

Zhu X. and Dale A. (2001). JavaAHP: a web-based decision analysis tool for natural resource and environmental management. Environmental Modelling and Software, 16(3) 251-262. 


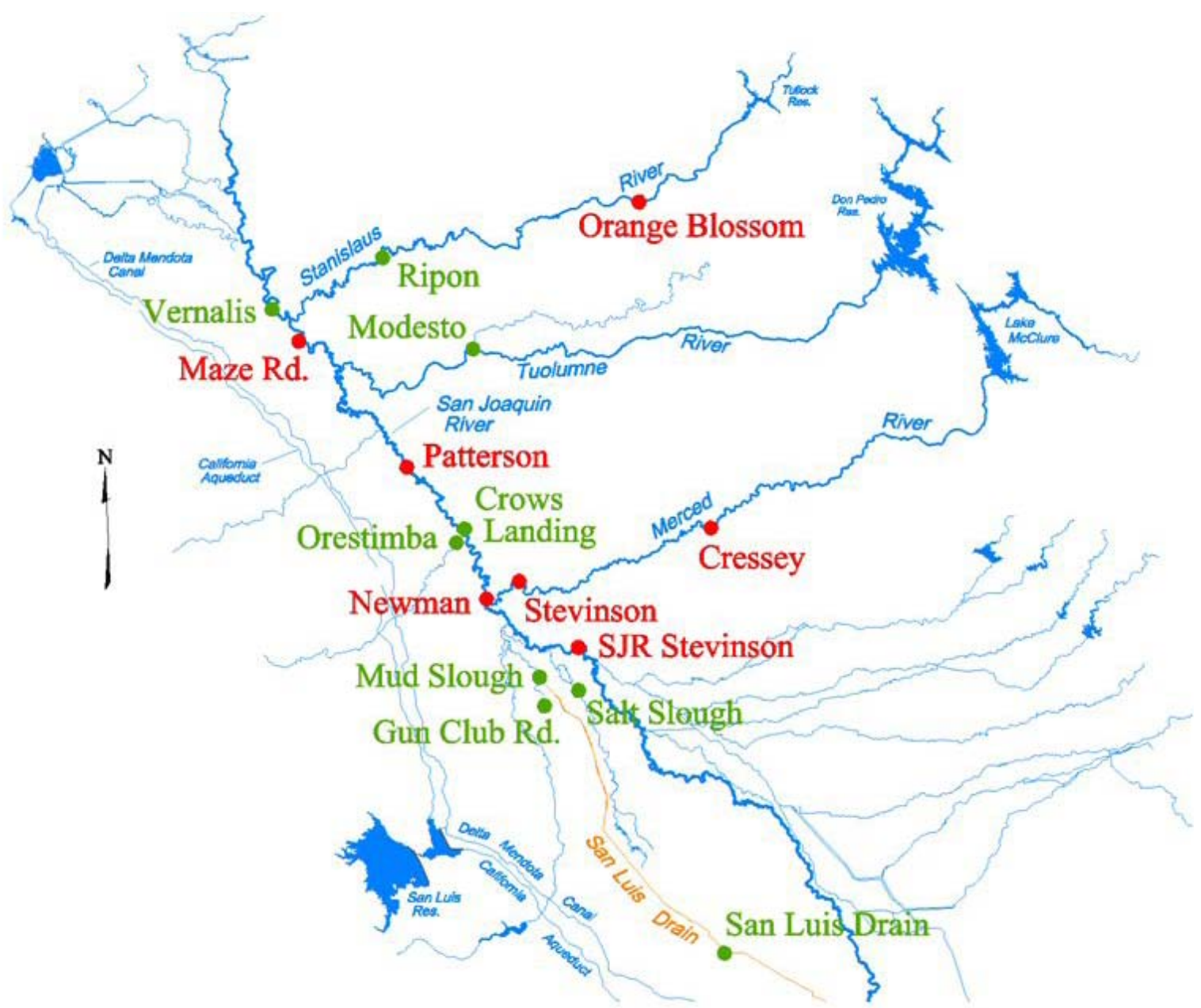

Figure 1. San Joaquin River Basin showing major primary real-time monitoring stations. The eastside of the Basin produces high quality return flows that derive from the snow pack in the Sierra Nevada mountains. The west-side of the Basin produces return flows high in salt and trace elements resulting from the combination of an imported water supply and the natural salinity of the alluvial, marine-derived soils (source: California Department of Water Resources). 


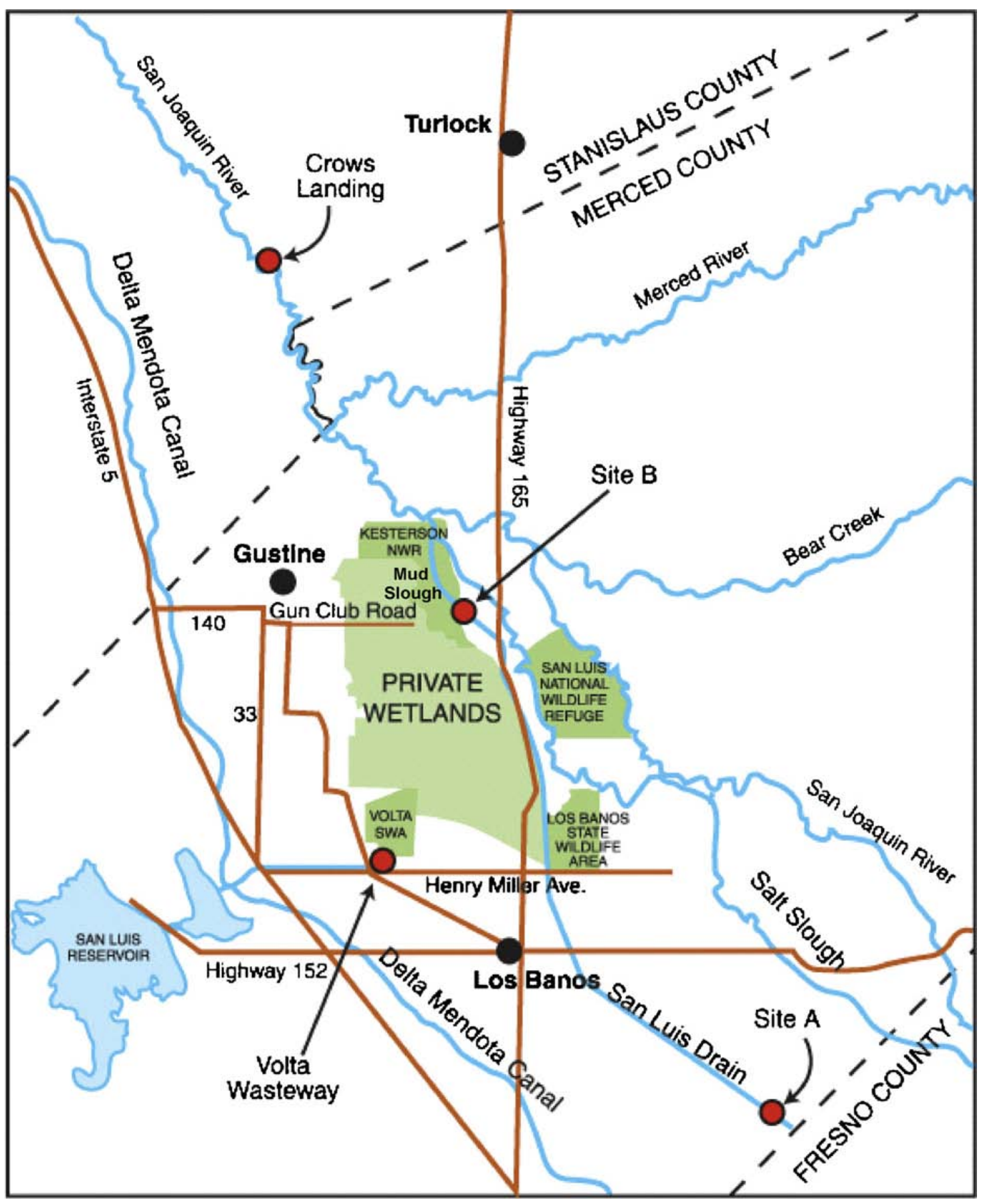

Figure 2. Location of federal, state and private wetlands that together comprise the 70,000 hectare Grasslands Ecological Area. Wetland drawdown in the spring months returns through Mud and Salt Sloughs to the San Joaquin River (Source: Bureau of Reclamation). 


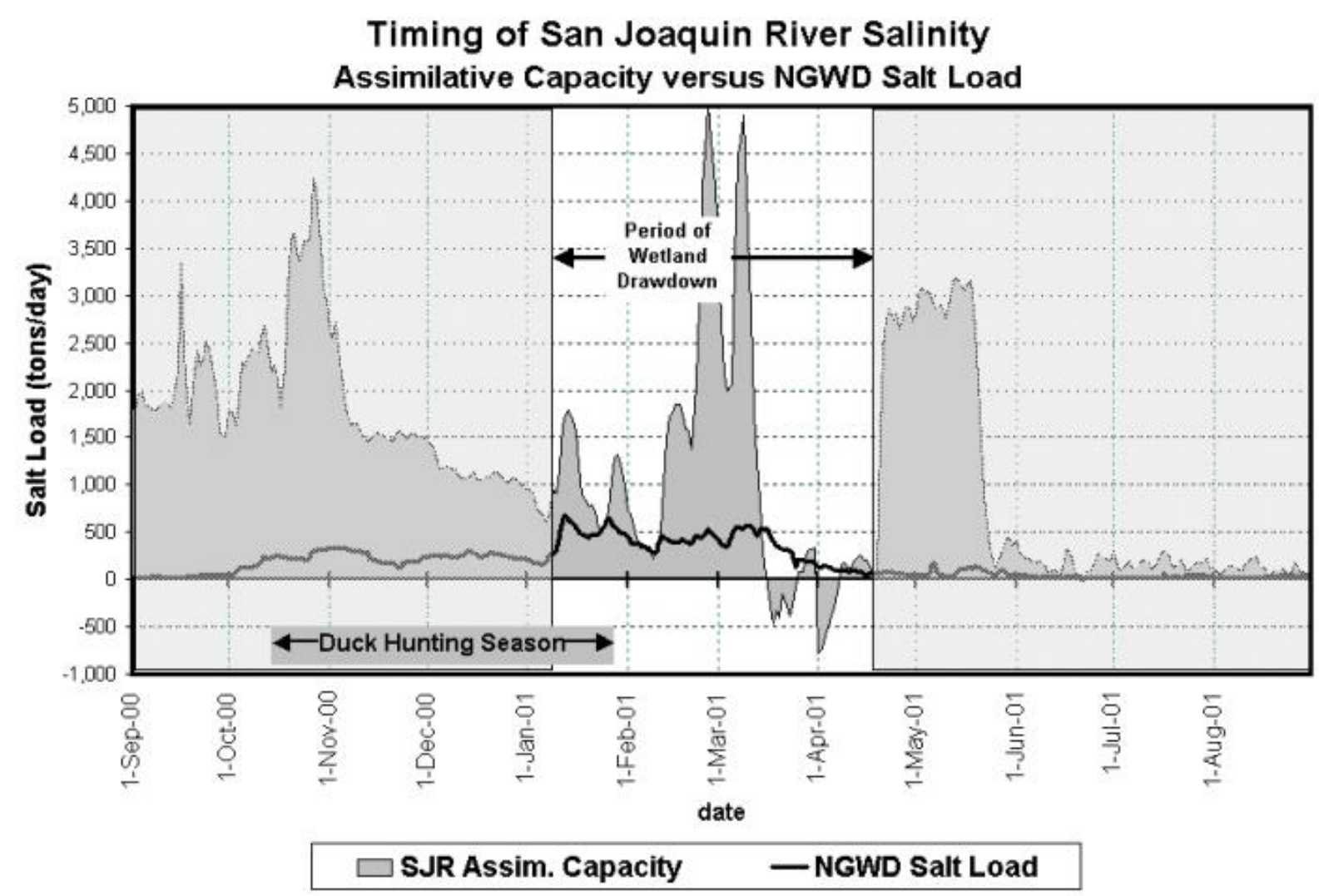

Figure 3. Illustration of the concept of real-time water quality management. Salt assimilative capacity (shown by the shaded grey area) is typically high during winter months when a combination of a higher salinity river objective $(1,000 \mathrm{uS} / \mathrm{cm})$ and rainfall runoff produce high diluting flows from east-side San Joaquin River tributaries. Wetland drainage salt loads from are sufficient to produce negative assimilative capacity during periods in late March and early April when river salt concentrations exceed State-mandated water quality objectives. Under a real-time salinity management scenario delaying wetland drainage drawdown until midApril would re-schedule this salt loading to a period of high River assimilative capacity preventing exceedence of river salinity objectives. 


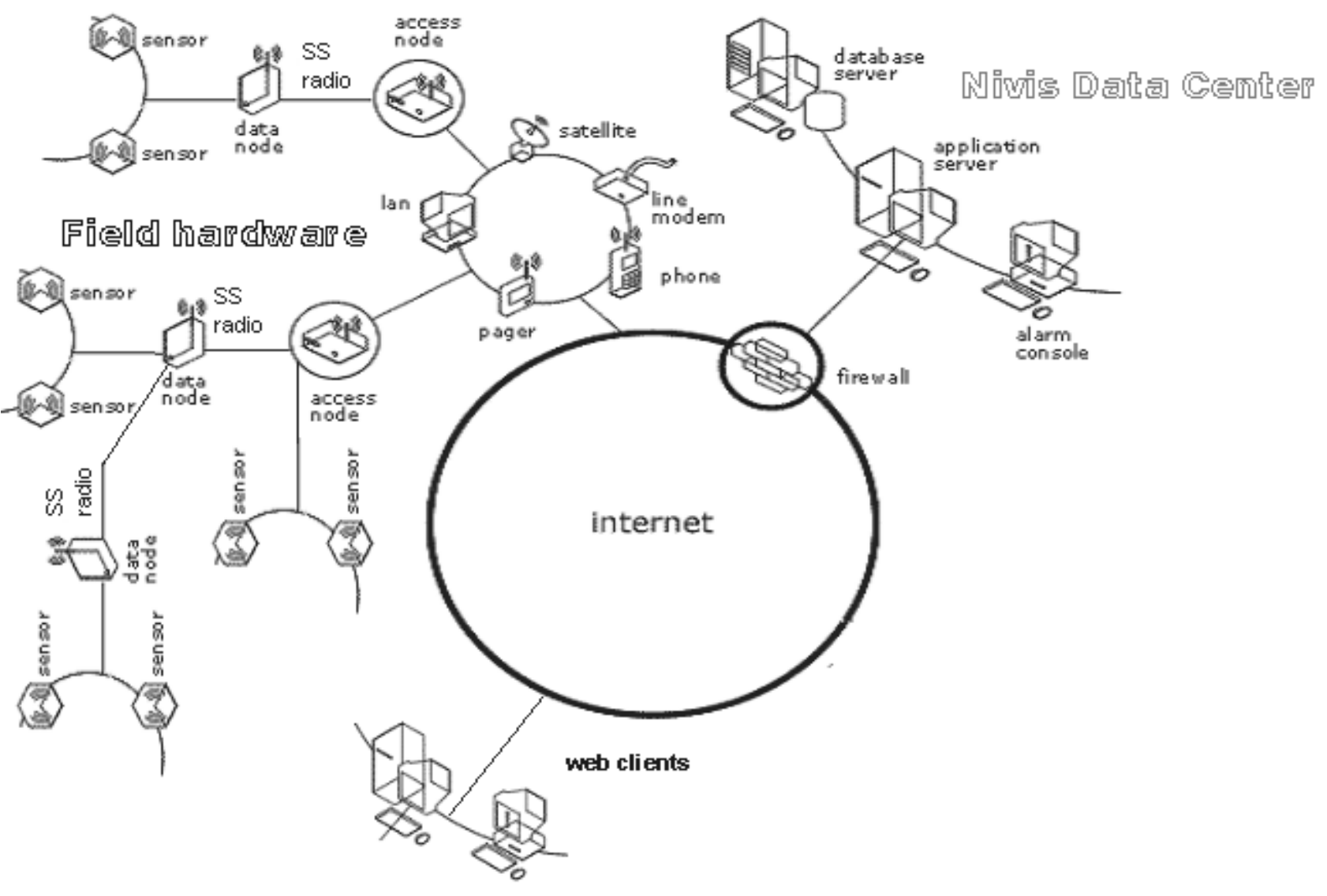

Figure 4. Environmental sensor network showing how individual sensors at each monitoring site report to local data nodes and communicate to each other through radio frequency telemetry. Master (access) nodes poll each data node and report data from multiple data nodes to the NIVIS data center. The NIVIS Data Center posts sensor data every 15 minutes to the web - allowing near real-time retrieval of preliminary data. Continuous data processing is required to produce data suitable for public access and retrieval (Source: YSI Inc.,2007). 




Figure 5. Data processing whiteboard within the Aquatic Informatics Aquarius software. Continuous environmental monitoring data is merged with weekly quality assurance data collected at each monitoring station to allow continuous error checking and data corrections to take place. Realtime water quality management (RTWQM) requires continuous data processing to allow daily salinity assimilative capacity forecasts to be made 




Figure 6. Protocol for replacing preliminary data on the NIVIS public website with data after quality assurance filtering has been completed. Public can download filtered historical data and has access to current sensor data. Private users have full access to both filtered and unfiltered data. 


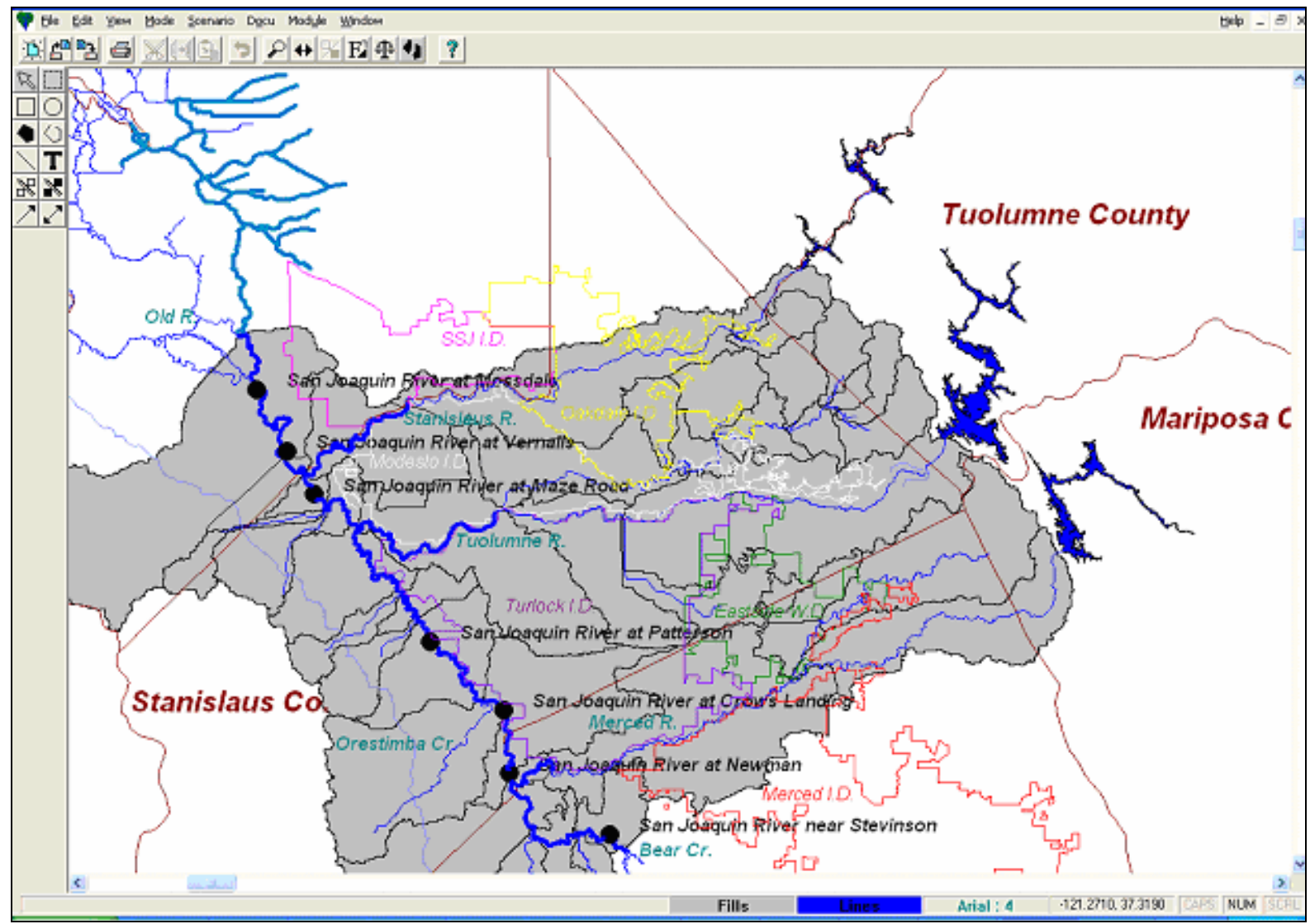

Figure 7. WARMF-SJR model representation of the main-stem of the San Joaquin River, California. Current continuous environmental monitoring stations are shown (as black circles) along the River. The WARM-SJR is a watershed-based decision support system which contains a comprehensive flow and water quality simulation model of the river, its major tributaries and the land use practices that contribute contaminant loads in surface and subsurface return flows to the River. The model is in the public domain and has been used to support TMDL planning activities in the US (Chen et al., 2001). 